\title{
Proposed criteria to determine whether a territory is free of a given animal disease
}

\author{
Barbara DuFOuR ${ }^{\mathrm{a} *}$, Régis PouILLOT $^{\mathrm{a}}$, Bernard TOMA $^{\mathrm{b}}$ \\ a Agence Française de Sécurité Sanitaire des Aliments (AFSSA), Unité d'Appui Épidémiologique \\ à l'Analyse de Risque, 23 avenue du Général de Gaulle, 94700 Maisons-Alfort, France \\ b École Nationale Vétérinaire d'Alfort (ENVA), Unité Pédagogique des Maladies Contagieuses, \\ 7 avenue du Général de Gaulle, 94700 Maisons-Alfort, France
}

(Received 16 December 2000; accepted 24 July 2001)

\begin{abstract}
This text describes the general principles underlying the concept of disease-free territory and the required statistical basis for the corresponding epidemiological surveillance operations. Among the essential points, it is emphasised that "disease-free" status should be given only under conditions substantiating the absence of infection (or infestation) and not simply on basis of a known low level of infection (or infestation). This manuscript also raises concerns about possible confusion that has arisen between the inevitable requirement to set a threshold on the level of detection of epidemiological surveillance tools, for economic reasons, and the acceptance of a level of infection (or infestation) that is known, but occurring below the accepted threshold, when according the official status of "territory-free from a given disease". In such a situation, it would be preferable to accord the status of "territory where the disease is in the process of eradication".
\end{abstract}

\section{free territory / infectious disease / prevalence rate threshold}

Résumé - Proposition de critères pour déclarer un territoire indemne d'une maladie animale. Ce texte rappelle les principes généraux sur lesquels devrait être fondée la notion de territoire indemne d'une maladie, ainsi que les bases statistiques nécessaires aux opérations d'épidémiosurveillance correspondantes. Parmi les éléments essentiels, les auteurs insistent sur la nécessité de ne conférer l'appellation « indemne de » que lorsqu'il n'est pas possible de prouver l'infection (ou l'infestation) et non pas lors de la présence certaine d'un faible taux d'infection (ou d'infestation). Ils attirent l'attention sur la confusion qui a dû s'installer entre l'inévitable exigence de fixer une limite au niveau de détectabilité des outils d'épidémiosurveillance, pour des raisons économiques, et l'acceptation d'un taux d'infection (ou d'infestation) connu, mais situé au-dessous de ce niveau, pour l'attribution officielle de la qualification « indemne de ». Pour une telle situation (prévalence faible, située au-dessous d'un seuil défini en fonction des caractéristiques épidémiologiques des maladies), l'appellation « territoire où la maladie est en voie d'éradication » est préférable.

territoire indemne / maladie infectieuse / prévalence limite acceptable

* Correspondence and reprints

Tel.: (33) 1497713 31; fax: (33) 14977 13 36; e-mail: b.dufour@afssa.fr 


\section{Contents}

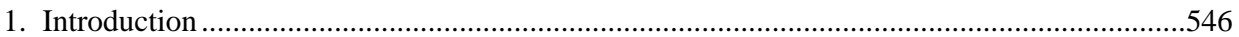

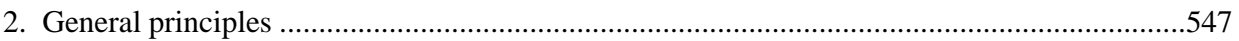

2.1. Total absence of the disease $v s$ situation when prevalence approaches zero.....................547

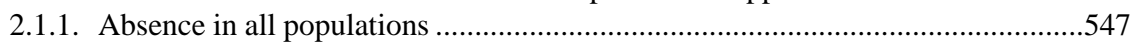

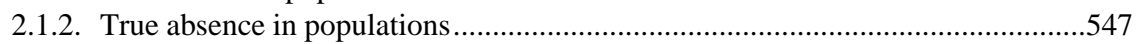

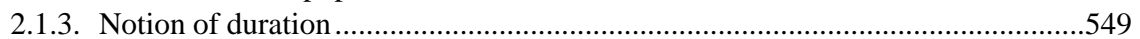

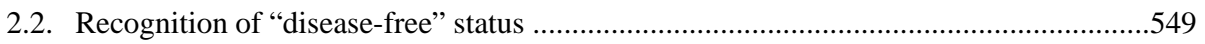

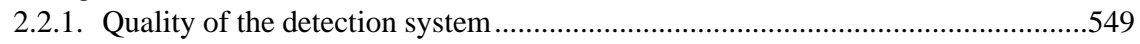

2.2.2. Territories with documented absence of disease or infection .............................550

2.2.3. Territories where the disease has previously occurred.......................................551

2.2.4. Appearance of the disease in a "disease-free" territory ......................................551

2.2.5. Long-term maintenance of disease-free status ................................................552

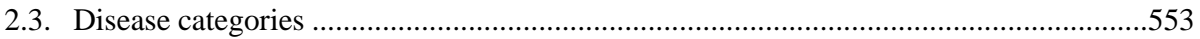

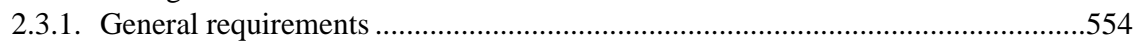

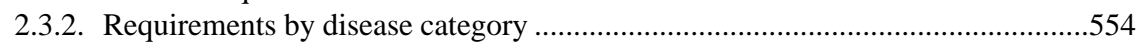

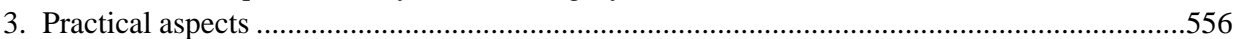

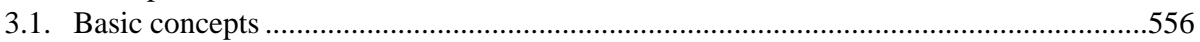

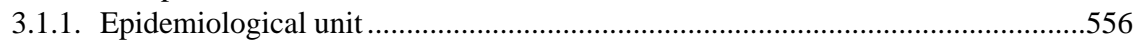

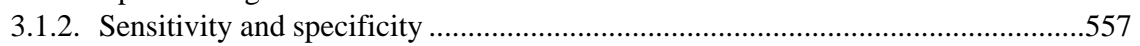

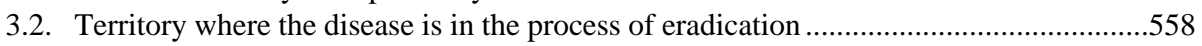

3.2.1. Defining the prevalence rate threshold...............................................................558

3.2.2. Determining how many animals are to be studied in each herd ...........................558

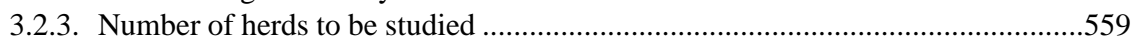

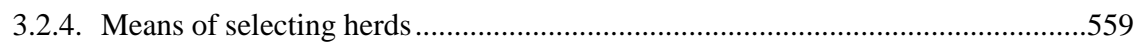

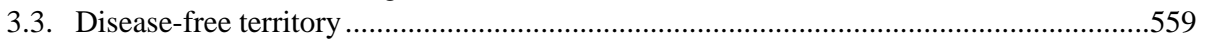

3.3.1. Existence of sub-populations at a higher level of risk..........................................560

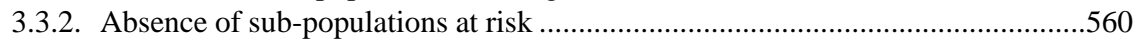

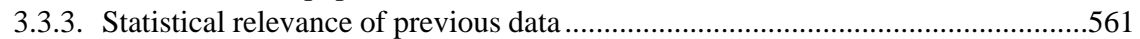

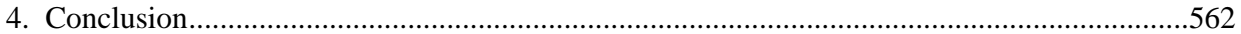

\section{INTRODUCTION}

The concept of disease-free territory for "some animal diseases" is important for several reasons. First of all, it is scientifically important, though it may be difficult to reach a consensus on the scientific definition of what constitutes a "disease-free territory", given the potentially wide range of views and opinions and the complexity of certain situations. Secondly, it is also important for economical and regulatory issues, since the adoption of a "legal" definition at the international level of a disease-free territory may have considerable economic repercussions on animals and animal products trade in this territory.

Several authors have proposed technical methods to define what constitutes a disease-free territory, either for specific diseases $[7,12,16,19]$ or in a broader range $[6$, $20,24,29]$. While these various approaches are necessary, a general review of the concept of disease-free territory with general principles that could be applied to all diseases is lacking.

This study deals only with the scientific approach to a definition of disease-free territory, leaving the responsibility for legal definitions to the competent international organisations. It is not a systematic review 
of publications in this field but rather a presentation of already known concepts and ideas and thoughts on this subject. The first section deals with general principles and general considerations on this subject. The second part deals with the practical aspects involved in the application of these principles.

\section{GENERAL PRINCIPLES}

\subsection{Total absence of the disease vs situation when prevalence approaches zero}

Before considering the criteria for deciding whether a territory is free of a disease, it is worthwhile summarising the various ways in which a pathogen may be present in a territory.

The pathogen may be carried (according to its pathogenic spectrum) by humans and/or domestic animals and/or wild vertebrates, and, depending on the case, may or may not be clinically expressed. It may also be present in arthropods or in the environment. A given pathogen may be present at the same time in several of these states. By giving each state a number, any situation can be represented by this criteria (Tab. I). Logically for a territory that is free from a given pathogen there would be no situation corresponding to numbers from 1 to 8 . This statement leads to two corollaries.

\subsubsection{Absence in all populations}

For a territory to be considered truly "free", the pathogen should not be present in vertebrates, arthropods or the environment. A territory with a formula including one or more number from 1 to 8 is not truly free in the full sense of the term.

This does not prevent terms being created that are strictly adapted to the situation (e.g. territory free of disease $\mathrm{X}$ in domestic animals), as opposed to the general term "disease-free" territory.

For example (Tab. I), the rabies in Denmark and in The Netherlands could be referred to as territories "free from rabies in domestic animals" or, better still, "free from rabies in terrestrial mammals" (i.e., not including rabies in Chiroptera or flying mammals).

\subsubsection{True absence in populations}

To qualify as "free", a territory one should not be aware of any case of the disease in question either clinically expressed or latent for a defined time frame.

Table I. Criteria taken into account to decide if a territory is free of the disease: example of diseases.

\begin{tabular}{lccccccccc}
\hline & 1 & 2 & 3 & 4 & 5 & 6 & 7 & 8 & Status \\
\hline Aujeszky disease in France [8] & no & no & yes & yes & yes & yes & no & no & No free \\
Plague (Yersinia pestis) in USA [3] & yes & no & yes & no & yes & no & yes & yes & No free \\
Rabies in Denmark & no & no & no & no & yes & (yes) & no & no & No free \\
African horse sickness in France & no & no & no & no & no & no & no & no & Free \\
\hline
\end{tabular}

1. Presence of the pathogen in humans, with symptoms.

2. Presence of the pathogen in humans, without symptoms.

3. Presence of the pathogen in domestic animals, with symptoms.

4. Presence of the pathogen in domestic animals, without symptoms.

5. Presence of the pathogen in wild animals, with symptoms.

6 . Presence of the pathogen in wild animals, without symptoms.

7. Presence of the pathogen in arthropods.

8. Presence of the pathogen in the external environment. 
This point needs to be considered in more details. For some diseases, especially those which are highly contagious (e.g. foot and mouth disease), everyone accepts the requirement of a total absence of outbreaks in order to qualify for "free" status. In contrast, for diseases that are only mildly contagious (e.g. enzootic bovine leukosis), free status is accorded by the Office international des Épizooties [22] (International Animal Health Code), when the prevalence of infection in animals or herds is low, namely below a maximum permitted prevalence rate (i.e., $0.2 \%$ or $1 \%$ ).

In this respect, two contrasting positions must be clearly distinguished when giving the status of disease-free territory:

- The first consists of giving "free" status despite the presence of a small number of outbreaks (clinical expression or latent infection) or a low prevalence rate of disease $X$ (below the maximum permitted prevalence rate) [2]. In this case, testing will be aimed at verifying whether or not the prevalence rate of the disease in the territory is below a predefined maximum permitted prevalence rate. The statistical problem (see below) is to determine the size of the sample needed to verify that the confidence interval corresponding to the estimated prevalence rate is entirely below the threshold. Nevertheless, in this case the presence of the disease at a prevalence rate below the maximum permitted level constitutes a permanent danger and includes the risk, proportionate to the degree of transmissibility of the pathogen, of an accidental flare-up of the disease and consequently of the maximum permitted prevalence being exceeded.

- The second consists of requiring the absence of any outbreaks of disease X (or infection). In this case, the problem is different: it is not generally feasible to test the entire susceptible animal population (domestic and wild). In most cases, testing for the presence of the disease is therefore based on sampling techniques. The aim is to detect as far as possible the existence of any outbreak of infection, while accepting the technical limits of sampling, which are influenced by economic constraints (cost of sampling and testing). This has two consequences:

- The first is that, unlike the previous case, the aim is not to establish the mean prevalence rate, but simply to detect any outbreaks of infection. Thus, to increase the probability of identifying any outbreak, testing should be carried out on a targeted sample (in other words a sample specifically targeted at high-risk groups) rather than a random sample of the general population at risk;

- The second is that the acceptable prevalence (i.e., the maximum prevalence rate of the disease that one accepts to detect with a confidence lower than a given threshold, e.g. 95 or $99 \%$ ), corresponding to random sampling of the overall population at risk, is chosen only for economic reasons (one cannot test the entire population), and that the detection of one or more outbreaks automatically precludes the status of "disease-free" being accorded.

The risk of confusion stemming from the existence of these two different concepts for attributing the status of disease-free territory is twofold.

- Firstly, between "maximum permitted prevalence rate" and "acceptable prevalence". These concepts are complex, and are not always easily distinguished. They encompass prevalence rates of the disease, for which the survey has a probability of detecting the existence of the disease by producing at least one positive response in a sample unit, that range from $5 \%$ (corresponding prevalence rate: maximum permitted prevalence rate) to 95\% (corresponding prevalence rate: acceptable prevalence) (see Fig. 1).

- Secondly, by confusing the minimum detectable prevalence applied for 


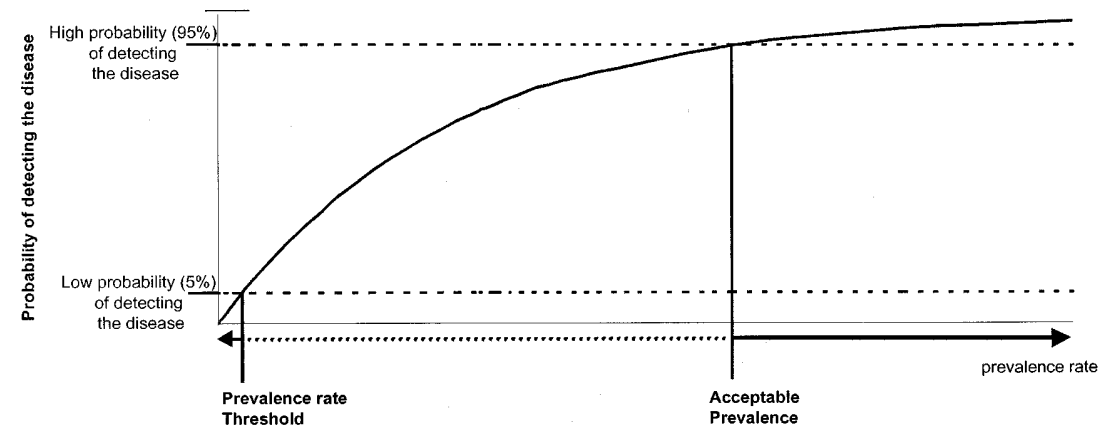

Figure 1. Diagram illustrating the concepts of prevalence rate threshold and acceptable prevalence.

statistical reasons with acceptance of the known presence of the disease at a prevalence rate below a maximum permitted level.

In practice, accepting a prevalence rate of $1 \%$ as the limit of detection of a sampling plan does not mean that one must consider as "disease-free" a territory where the herd prevalence rate is $0.5 \%$.

\subsubsection{Notion of duration}

The complete absence of the pathogen, a necessary condition to qualify for the status of disease-free territory, must be associated with the notion of the period of time during which the disease must have been absent.

This period, which will vary according to the characteristics of the disease in question, is highly dependent on the incubation period of the disease and whether or not there could be asymptomatic carriers.

Thus, for a disease with a short incubation period and which is rarely or never expressed in subclinical form or as latent infection (i.e., healthy carrier status), there is no need to wait long after the apparent disappearance of outbreaks: for example, transmissible gastroenteritis in pigs.

In contrast, for a disease with a long incubation period and/or a high rate of healthy carriers, the required waiting period after the last observed case of the disease will necessarily be long: for example, Aujeszky's disease, bovine spongiform encephalopathy and scrapie.

\subsection{Recognition of "disease-free" status}

The previously existing status of a territory wishing to be recognised as free from a disease is either that of a country where the disease had never been identified or that of a country where the disease had been identified and controlled.

We shall consider these two situations in turn. However, we must first consider the quality of the system used to detect the presence of the disease.

\subsubsection{Quality of the detection system}

Identification of the presence of an animal disease depends on the efficacy of the Veterinary Services and the quality of the epidemiological surveillance for the disease [15]. Semi-quantitative evaluation systems have been proposed for each of these elements $[9,14]$. The probability that a disease is truly absent from a territory is greater when the scores for each of the two evaluations are high. It is advisable to take into 
account the results of these evaluations during the process of qualifying a territory as "disease-free". This could be done either:

- by adapting the period during which epidemiological surveillance sampling must be carried out, depending on the results of these evaluations (the lower the evaluation scores the longer the period before attributing "disease-free" status); or

- by adapting the required number of sampling units, according to the same principle (the lower the evaluation scores the higher the number of samples); or

- by a combination of these two factors.

\subsubsection{Territories with documented absence of disease or infection}

Less stringent requirements may be acceptable for substantiating the diseasefree status of territories where a specific disease has never been recorded (or has not been recorded for a defined period of time), than for attributing disease-free status to a territory where the disease has been identified, been controlled and apparently been eradicated.

Examples of such situations are numerous, especially for diseases that require special ecological conditions in order to be transmitted by vectors: thus, it may be assumed that the countries of northern Europe are free from various tropical arboviral diseases: African horse sickness, yellow fever, etc. [1, 4] (Tab. II).

Furthermore, for all diseases that require a vector (a single species or only a small number of species) for transmission, it can be assumed that the absence of the vector(s) in the concerned territory is sufficient to allow the territory to be recognised as "historically free". This is, for example, the case for France, a territory free of yellow fever (absence of Aedes aegypti). However, only a small proportion of diseases fulfils this description (i.e., require a vector).

Table II. Diseases usually occurring in epizootic form.

\begin{tabular}{|c|c|c|c|}
\hline Soil-borne & Wild animals & Arthropods & \\
\hline no & no & no & $\begin{array}{l}\text { Sheep pox } \\
\text { Equine influenza } \\
\text { Swine vesicular disease }{ }^{\text {a }} \\
\text { Peste des petits ruminants } \\
\text { Rabbit haemorrhagic disease }^{\text {a }} \\
\text { Rinderpest }^{\text {a }}\end{array}$ \\
\hline no & yes & no & $\begin{array}{l}\text { Avian influenza } \\
\text { Foot and mouth disease } \\
\text { Classical swine fever } \\
\text { Aujeszky's disease } \\
\text { Newcastle disease }\end{array}$ \\
\hline no & yes & yes & $\begin{array}{l}\text { African swine fever } \\
\text { African horse sickness } \\
\text { Plague (Yersinia pestis) } \\
\text { Bluetongue } \\
\text { Rift Valley fever } \\
\text { Equine viral encephalomyelitis } \\
\text { Myxomatosis } \\
\text { Vesicular stomatitis }\end{array}$ \\
\hline
\end{tabular}

\footnotetext{
${ }^{a}$ In rare or exceptional circumstances wild animals may also be affected.
} 
For all other diseases, it is logical to accept less stringent requirements recognising such a territory as "disease-free" compared to the requirements for a previously infected territory, as long as the evaluation scores for the Veterinary Services and the system of epidemiological surveillance are taken into account. To return to the aforementioned proposal to adapt requirements according to the evaluation results (for scores above a certain minimum level), this could be applied in proportion to the scores. Below the minimum levels no adjustments would be made.

For according the status of "territory historically free from disease X", it may not be necessary to define separate conditions for each disease $\mathrm{X}$ : diseases could first be classified using basic criteria, and requirements could then be defined for each disease category (see below).

\subsubsection{Territories where the disease has previously occurred}

Evidence must be provided that the disease has really disappeared.

- The first step is to define the status that is being sought. Does the status of "territory free from disease $X$ " imply a total absence of the pathogen, or more simply "territory free from disease $\mathrm{X}$ in domestic animals" (e.g. where there is a wildlife reservoir)?

- Secondly, one must avoid the unclear situation of attributing free status to a territory that continues to vaccinate against the disease that has "disappeared". Irrespective of the situation, the term "territory free from disease X" should never be applied to a territory where vaccination continues to be used against disease. The reason is clear: for many diseases, vaccination and testing are incompatible; and in those cases where they are compatible (e.g. Aujeszky's disease [26]) the existence of carriers, even if they have been vaccinated, introduces an additional risk compared to a territory using only sanitary measures. Of course, this is not intended to decry the use of vaccination, where this is judged necessary in order to control the animal health situation (France, like many other west European countries, continued to vaccinate against foot and mouth disease for 10 years after the last outbreak [21]) but is simply aimed at avoiding confusion. When vaccination continues to be used, at least for a certain period, it is perfectly reasonable to apply the concept of "territory where disease $\mathrm{X}$ is in the process of eradication", as an intermediate stage between "infected territory" and "disease-free territory". It should be possible to apply the term "territory where disease $\mathrm{X}$ is in the process of eradication" if the prevalence rate of the disease is below a maximum permitted prevalence rate, determined according to the characteristics of the disease.

- Lastly, the system of detection for the disease in question should be applied during the required period, respecting the permitted prevalence rate established for the relevant category of disease (see below).

As in the case of territories that are historically free from a disease, requirements can be adapted to different categories of disease.

\subsubsection{Appearance of the disease in a "disease-free" territory}

At any moment, a disease that has "disappeared" from a territory (either truly or only apparently) can reappear. One should not lose sight of the fact that the status "disease-free" corresponds to a temporary situation, which is based on "retrospective" facts (the facts used to that classification are restropective), and which, although it has a prospective significance (the probability of disease outbreaks occurring in a diseasefree territory is lower than in an infected 
territory), it does not provide an absolute warrantee of future absence of the disease.

Various mechanisms can be responsible for a reappearing disease:

- a resurgence of a disease may occur as a result of the pathogen persisting in one of the susceptible populations in a territory or in the external environment;

- the pathogen may be reintroduced into a territory by one or more of many possible vectors: imported domestic or wild animals, cross-border contacts between domestic animals, movements of wild animals, importation of foodstuffs or products of animal origin, etc.

The probability of the disease being introduced is inversely proportional to the level of precautionary measures, suitably adapted to the "epidemiological type" of the disease, and to the epidemiological status of the neighbouring territories and/or territories with which it conducts trade.

The reappearance of the disease can lead to either of the following two situations, depending on the disease:

- For a classic transmissible disease (bacterial disease, viral disease, etc.), the outbreak(s) will result in the loss of the territory's free status, and a return to the category "territory where the disease is in the process of eradication", particularly if tracing back reveals shortcomings (inadequate surveillance, permeability of frontiers, etc.). In special cases, where the epidemiological investigation unequivocally shows the absence of any transmission beyond the outbreak, the "free" status might simply be suspended for the time it takes to eradicate the outbreak, disinfect and ensure depopulation before any reintroduction of animals, and then be reinstated;

- For a situation corresponding to an epidemiological dead-end, the occurrence of an outbreak should not lead to the suspension of "free" status, if there is a clear explanation for the origin of the case(s).

\subsubsection{Long-term maintenance of disease-free status}

It is logical to accept that the surveillance procedures for a given disease are not identical in a territory that has just acquired "disease-free" status and in a territory that has enjoyed this status for many years.

The essential difference between these two situations concerns the risk of "resurgence". In a territory that has been diseasefree for a long time, one cannot exclude the hypothesis that the pathogen persists unrecognised in some individuals. In contrast, in a territory that has been "diseasefree" for a long time, the only risk is that the pathogen may be reintroduced. In the latter case, the surveillance procedures should be strictly adapted to the nature of the risks of reintroduction and (independently of measures taken to reduce these risks) should be based on samples defined in accordance with these risks and not by using randomly-selected samples for the entire susceptible population.

- For epizootic diseases, with a high rate of clinical expression, a system of epidemiovigilance should be permanently maintained, with the capacity to handle any suspected cases that may appear and to confirm or invalidate them in a specialised laboratory. Surveillance by sampling, however, would not appear to be indispensable.

- For diseases that are enzootic in character or frequently cause subclinical infection, once the first few years have passed without any resurgence of the disease, epidemiological surveillance should be maintained specifically targeting the risks of a reintroduction of the pathogen (based on risk analysis results). This should normally be covered by the sanitary measures applied to the importation of animals and animal products.

It may also be useful to consider two general principles regarding the relationship 
between epidemiological surveillance and preventive measures:

- The first principle is that the higher the risk of reintroducing the disease, as determined by the results of epidemiological surveillance (existence of a potential wildlife reservoir, etc.), the higher the level of preventive measures that should be applied.

- The second principle is that for a given level of risk of reintroduction, the higher the level of preventive measures, the less intensive the epidemiological surveillance measures need to be.

\subsection{Disease categories}

The aim of defining categories of diseases is to group together diseases that have similar epidemiological and pathological characteristics and may therefore be subject to similar requirements regarding the verification of their absence and the attribution of the status of "free" territory.

- The first criterion is the usual epidemiological type of the disease, thus differentiating between diseases that are normally expressed in the form of epizootics and those that are enzootic in character.

A major difficulty encountered is the lack of a clear boundary between epizootic and enzootic, and the possibility that a given disease may sometimes pass from one category to the other. Apart from this major reservation, most diseases fall clearly into one or other of the epidemiological types (see Tabs. II and III).

- The second criterion is the range of populations (or types of environment) where the pathogen can reproduce and/or persist. The range of susceptible species (or environments where the pathogen can persist) directly influences the size of the populations to be included in the

Table III. Diseases usually occurring in enzootic form.

\begin{tabular}{llll}
\hline Soil-borne & Wild animals & Arthropods & \\
\hline yes & yes & no & Anthrax a \\
& & Melioidosis \\
no & yes & yes & Tularaemia \\
& & Plague due to Yersinia pestis \\
& yes & no & Rabies \\
no & no & Enzootic bovine leukosis \\
no & & Contagious bovine pleuropneumonia \\
& & & Bovine tuberculosis b \\
& & Scrapie \\
& & Infectious bovine rhinotracheitis \\
& & Teschen disease \\
& & Bovine spongiform encephalopathy \\
& & Bovine brucellosis \\
& & Caprine and ovine brucellosis \\
& & Contagious caprine pleuropneumonia \\
& & Dourine \\
& & Glanders \\
& &
\end{tabular}

In rare or exceptional circumstances arthropods ${ }^{\mathrm{a}}$ or wild animals ${ }^{\mathrm{b}}$ may also be involved. 
epidemiological surveillance operations and thus the extent of the preventive measures.

We propose four categories of species or environment to be taken into account: domestic animals, wild animals, arthropods and the external environment.

As with the previous criterion, difficulties can be encountered in attempting this classification, insofar as a given disease may, depending on the place and time, change its type of host and undergo several modes of transmission (e.g. with or without biting arthropods, such as African swine fever which, according to the existing conditions, uses one or other mode of transmission) [23]. However, as with the previous criterion, the behaviour of a disease in a given environment is in a large majority of cases determined in a preferential manner (see Tabs. II and III).

Given the variations encountered for each of the two proposed criteria, the groups thus formed should not be taken as hard and fast, but rather as a guide should in determining the level of requirements for the status of "free" territory.

For a territory to qualify for disease-free status, one can define general requirements, applicable whatever the disease, territory or date, and specific requirements by category of disease.

\subsubsection{General requirements}

Five general requirements are proposed and should be considered as essential:

- Absence of any outbreaks of disease X, expressed clinically or subclinically (i.e., healthy carriers), in any susceptible population in the territory. This requirement means that a territory in which outbreaks of the disease are still being observed should not receive the status of diseasefree territory. In such cases, the term "territory where disease $\mathrm{X}$ is in the process of eradication" is appropriate. It also means that neither should a territory where the disease has been eradicated in domestic animals but persists in wild animals be accorded the status of disease-free territory. In such cases, the situation should be conveyed by a term along the lines of "territory free of disease $\mathrm{X}$ in domestic animals".

- The disease should be officially notifiable. This second requirement reduces the risk of the animal health authorities remaining unaware of any occurrence or recurrence of the disease. All suspected cases must be brought to their attention.

- Absence of vaccination against the disease. The third requirement has already been commented on. It is useful at this point to emphasise the danger in using the term (and the concept) "territory-free from disease $\mathrm{X}$ where vaccination is practised".

- System of epidemiological surveillance based on sampling and the capacity to study suspected cases rapidly in the laboratory. The fourth requirement relates to the capacity of the territory to exercise appropriate surveillance for the referred disease. Depending on the case, this may involve random sampling or targeted sampling, or indeed a system adapted to the detection of any suspected cases of the disease in question and laboratory evidence that the pathogen responsible for the disease is not present.

- Preventive measures applicable to the importation of "commodities" (in the broad sense of the term, including live animals). The latter requirement is legitimate in that it is aimed at reducing the risk of introducing the disease into a territory that is free of the disease.

\subsubsection{Requirements by disease category}

- Soil-borne diseases: the model for this category is anthrax [17] (see Tab. III). Taking into account the existing epidemiological knowledge of this disease, it would be impossible to propose a 
status of "territory free from anthrax" for a territory where the disease has already occurred, regardless of the date.

- Arthropod-borne diseases: if one or more species of arthropod vector(s) exist in the territory in question, the disease falls within the general category. On the other hand, if it can be proved that no vector species exists in the territory, the latter can be considered as a territory free from the corresponding disease. The accidental importation of an infected (or infested) individual should not jeopardise this status.

- Epizootic diseases: by definition, epizootic diseases tend to occur in the form of numerous outbreaks and, in most cases, with noticeable clinical expression, at least in domestic animals, and even in wild animals. Once all vaccination against a disease of this type has ceased, and the entire population has become fully susceptible again, any persistence of the pathogen in this population would be more easily detected than that of the disease agent of an enzootic or sporadic type of disease with a high level of latent infection. This leads to the following corollaries: firstly, surveillance can be limited to a systematic investigation of any suspected cases, without necessarily having recourse to sampling; secondly, the waiting period after the last outbreak has been detected can be a little shorter than for enzootic diseases, which frequently occur in a latent form.

A reasonable waiting period after the last outbreak has been observed and all vaccination against the disease has ceased would be around 2 to 3 years.

If sampling is carried out, the results must be interpreted taking into account the risk of finding post-vaccinal or post-infectious residual antibodies. This depends on the date that vaccination ceased, the length of useful life of the animal species in question, the kinetics of post-vaccinal antibodies and the characteristics of the test used.
This is of course valid for diseases that occur in domestic animals, without a wildlife reservoir. This is notably the case with sheep pox and equine influenza (see Tab. II).

For epizootic diseases where there is a potential wildlife reservoir, the status of these animals must be verified before attributing the status of disease-free territory (examples: classical swine fever, Aujeszky's disease) [18]. This will inevitably be more difficult than epidemiological surveillance for the disease in domestic animals.

Some epizootic diseases can take on an enzootic character, especially in tropical regions, with only a limited amount of clinical expression: African swine fever, African horse sickness, bluetongue, etc. Thus the absence of vaccination and any identified clinical cases is not in itself sufficient evidence for a territory to be considered disease-free. Sampling in domestic and/or wild animals may reveal that the virus is still circulating.

- Enzootic diseases: when these diseases are circulating in wild animals, it can be difficult to succeed in attributing the status of "disease-free territory". The disease may develop in certain wild animal species without any occurrence in humans or in domestic animals, especially with small wild animals such as rodents. The general requirements are thus insufficient, as for example in the case of human plague (caused by Yersinia pestis) [3]. In some regions, the disease may persist and develop without affecting humans or domestic animals and is only detected accidentally, notably through a human case (in some States in the United States of America for example).

Enzootic diseases require regular active epidemiological surveillance through sampling, either solely in domestic species (e.g. enzootic bovine leukosis) when wild animal species are not susceptible (or only exceptionally so), or in both domestic and wild animals (e.g. rabies [5]). In domestic 
animals, the characteristics of the survey must be adapted to the species affected and to the disease.

For the survey, two maximum permitted prevalence rates are often proposed in the literature: $1 \%$ of herds and $0.2 \%$ of herds. The most frequently used probability of detecting the disease is either $95 \%$ or $99 \%$. It should be borne in mind that:

- the disease may be detected even if the prevalence rate of infected herds is markedly lower than the chosen maximum permitted prevalence rate;

- at these prevalence rates, the probability of the disease not being detected is $5 \%$ and $1 \%$, respectively;

- the cost of sampling participates to determine how low the permitted prevalence rate can be set;

- the length of the waiting period before "disease-free" status can be accorded should be inversely proportional to the chosen probability of detection $(95 \%$ or 99\%);

- if sampling is repeated annually and the results are negative, this tends to increase the probability that the disease is absent;

- if any outbreaks are detected, a new waiting period will have to be imposed before disease-free status can be accorded (and in the case of a disease-free territory the status will have to be reassessed).

In practical terms, for this epizootic disease, the period that we propose during which the results of epidemiological surveillance should have been negative is about 5 years (for example) for a protocol with a $95 \%$ probability of detecting a herd infection rate of $1 \%$ (with a within-herd prevalence rate of $\geq 5 \%$ ), 2 years for a protocol with a $99 \%$ probability of detecting a herd infection rate of $0.2 \%$ (with a within-herd prevalence rate of $\geq 5 \%$ ), or any intermediate modality between these two proposals. This pragmatic approach to fixing the period must also take into account the stability of the epidemiological status of the disease in the territory in question.

- Sporadic diseases: the general requirements remain valid. However, a disease that occurs sporadically is often associated with a long incubation period, making it very difficult to apply a system of epidemiological surveillance. This is particularly the case for bovine spongiform encephalopathy (BSE), which raises specific problems over the definition of requirements for according the status of "BSE free".

\section{PRACTICAL ASPECTS}

As we saw in Section 1, two main questions have to be answered, both requiring the use of statistical tools. Chronologically, the first question relates to a territory known to be still infected, but with a low prevalence rate, and wishing to be accorded the status of "territory where disease $\mathrm{X}$ is in the process of eradication": "Is the herd prevalence rate below a given level?" The corollary questions are as follows: (a) "How many herds must be investigated?" and (b) "How should these herds be selected?" The second question concerns a territory that already enjoys the status of "territory where disease X is in the process of eradication", or wishes to obtain directly the status "territory free from disease X": "Is the disease (still) present in the territory?"

\subsection{Basic concepts}

\subsubsection{Epidemiological unit}

For a number of diseases, epidemiological surveillance must take into account both domestic and wild animal populations:

- For most categories of domestic animals the epidemiological unit most commonly used is the herd (or flock), and the 
measure of disease frequency will therefore be the herd prevalence rate;

- For wild animals the concept of herd as applied to livestock production (namely an identified group of animals living permanently together with little or no interaction with other groups of the same species) cannot be readily applied. The preferred epidemiological unit is therefore the individual animal, and the measure of disease frequency will therefore be the prevalence rate of infected animals.

\subsubsection{Sensitivity and specificity}

The number of units that have to be tested is usually provided by tables that assuming the sensitivity and specificity of the dichotomous procedure that allow to declare each unit to be "infected" or "disease-free" to be equal to 1 (e.g. [27]), despite the fact that very few tests actually have this characteristic.

When the epidemiological unit is the animal, it is the individual sensitivity and specificity of the test that must be taken into account. When the epidemiological unit is a group, an evaluation of the diagnostic value of testing over the entire territory requires two levels of analysis: one at the level of the epidemiological unit, and the other at the level of the territory.

At the level of the epidemiological unit the sample may be exhaustive or partial, in other words all or only some of the animals in each herd are tested. In this case, we must define the mean within-herd level of prevalence that would need to be detected with a high probability (95\% or $99 \%$ ). This determines the number of subjects in each epidemiological unit that will have to be tested, according to the number of animals in the unit (Tab. IV), and as a corollary, the mean herd-level sensitivity ( $95 \%$ or $99 \%$ ).

This reasoning becomes more complex when the sensitivity and specificity of the survey test are not equal to 1 . This point has been studied in the veterinary field for a number of years [20] and computer programs are now available to evaluate the "herd" diagnostic value for all the cases observed [10, 11, 13].

For almost all diseases, it is reasonable to admit that, in practice, the procedure used will offer a perfect herd-level specificity: a combination of clinical, epidemiological, serological, histological and/or bacteriological confirmatory procedures will allow the decision maker to declare an epidemiological unit as infected only on the basis of true infected epidemiological unit.

The epidemiological unit sensitivity must be accurately estimated. The required number of epidemiological unit given by the classical tables (e.g. [27] or Tab. IV) for a test with a sensitivity of $1(100 \%)$, will need to be modified according to the epidemiological unit sensitivity. A practical approximation is to use the apparent prevalence concept. If the aim is to be able to detect a true prevalence of $T P=1 \%$ with a test having a sensitivity of $S e=90 \%$ and a specificity of $100 \%$, a procedure will have to be used that is capable of detecting an apparent prevalence of approximately $T P \times S e=$ $0.01 \times 0.9=0.9 \%$. Note that in the following part, the term "true prevalence" will be used. Reader should interpret it as "apparent" or "true" prevalence depending on his own context.

The estimation of individual and herdlevel sensitivity and specificity is of difficult concern. Since the diagnostic values of the tests may be very variable according to the local context, the figures given in the tables shown below should therefore not be applied too strictly or mathematically. These figures merely give an idea of the size needed to attain a given level of precision.

Having introduced the basic concepts, we shall now consider the answers to the two questions. 
Table IV. Number of subjects needed to detect a disease with a probability of $95 \%$ (A) or $99 \%$ (B) as a function of population size and the level of prevalence to be detected, assuming a test sensitivity and specificity equal to 1 .

A

\begin{tabular}{|c|c|}
\hline Size & Prevalence rate to be detected (\%) \\
\hline & $\begin{array}{llll}2 & 5 & 8 & 10\end{array}$ \\
\hline
\end{tabular}

\begin{tabular}{rccccccccccc}
\hline 10 & 10 & 10 & 10 & 10 & 10 & 9 & 8 & 7 & 6 & 5 & 4 \\
20 & 20 & 20 & 19 & 17 & 16 & 13 & 10 & 9 & 7 & 6 & 5 \\
50 & 50 & 48 & 35 & 26 & 22 & 16 & 12 & 10 & 8 & 6 & 5 \\
100 & 95 & 78 & 45 & 31 & 25 & 17 & 13 & 10 & 9 & 6 & 5 \\
200 & 155 & 105 & 51 & 33 & 27 & 18 & 14 & 11 & 9 & 6 & 5 \\
300 & 189 & 117 & 54 & 34 & 28 & 18 & 14 & 11 & 9 & 6 & 5 \\
400 & 211 & 124 & 55 & 35 & 28 & 19 & 14 & 11 & 9 & 6 & 5 \\
500 & 225 & 129 & 56 & 35 & 28 & 19 & 14 & 11 & 9 & 6 & 5 \\
600 & 235 & 132 & 56 & 35 & 28 & 19 & 14 & 11 & 9 & 6 & 5 \\
700 & 243 & 134 & 57 & 36 & 28 & 19 & 14 & 11 & 9 & 6 & 5 \\
800 & 249 & 136 & 57 & 36 & 28 & 19 & 14 & 11 & 9 & 6 & 5 \\
900 & 254 & 137 & 57 & 36 & 29 & 19 & 14 & 11 & 9 & 6 & 5 \\
1,000 & 258 & 138 & 57 & 36 & 29 & 19 & 14 & 11 & 9 & 6 & 5 \\
2,000 & 277 & 143 & 58 & 36 & 29 & 19 & 14 & 11 & 9 & 6 & 5 \\
3,000 & 284 & 145 & 58 & 36 & 29 & 19 & 14 & 11 & 9 & 6 & 5 \\
4,000 & 288 & 146 & 58 & 36 & 29 & 19 & 14 & 11 & 9 & 6 & 5 \\
5,000 & 290 & 147 & 59 & 36 & 29 & 19 & 14 & 11 & 9 & 6 & 5 \\
Infinity & 299 & 149 & 59 & 36 & 29 & 19 & 14 & 11 & 9 & 6 & 5
\end{tabular}

\section{B}

\begin{tabular}{|c|c|c|c|c|c|c|c|c|c|}
\hline \multirow{2}{*}{$\begin{array}{l}\text { Size } \\
\text { of the } \\
\text { population }\end{array}$} & \multicolumn{9}{|c|}{ Prevalence rate to be detected (\%) } \\
\hline & & 2 & 5 & 81 & 1015 & 20 & 25 & 0 & \\
\hline & & & 10 & & 1 & 0 & 88 & & \\
\hline & & & & & 18 & 513 & 11 & & \\
\hline & & & 423 & & $\begin{array}{lll}29 & 2\end{array}$ & 217 & 141 & & \\
\hline & & & 59 & 433 & $\begin{array}{lll}36 & 2\end{array}$ & 519 & 15 & & \\
\hline & & & 73 & 494 & 40 & 720 & & & \\
\hline & & & 785 & & 41 & 0 & & & \\
\hline & & & 815 & 524 & 42 & 21 & & & \\
\hline & & & 83 & 534 & 42 & 321 & & & \\
\hline & & & 4 & 534 & 43 & 321 & & & \\
\hline & & & 8. & 544 & & 21 & & & \\
\hline & & & 8 & & & & & & \\
\hline & & & 86 & 544 & 43 & 21 & & & \\
\hline & & & 86 & 544 & 43 & 21 & & & \\
\hline & & & 88 & 554 & 442 & 9 21 & & & \\
\hline & & & 89 & 554 & 44 & 21 & & & \\
\hline & & & . & 554 & & & & & \\
\hline & & & 2 & 554 & 44 & & & & \\
\hline & & & & & & & & & \\
\hline
\end{tabular}

\subsection{Territory where the disease is in the process of eradication}

Is the prevalence rate of infected herds (domestic animals) in the territory below a prevalence rate threshold? To answer this question, we must first:

- define the prevalence rate threshold;

- determine how many herds are to be studied;

- decide how to select these herds;

- determine how many animals are to be studied in each herd.

\subsubsection{Defining the prevalence rate threshold}

Defining the prevalence rate threshold is an arbitrary though fairly logical process.
Parameters like economical, sanitary and social consequences must be taken into account, as well as biological profile of the disease itself.

\subsubsection{Determining how many animals are to be studied in each herd}

First, the intra-herd prevalence rate that one wishes to be able to detect in $p=95 \%$ or $p=99 \%$ of cases has to be established, and then the number of animals to be studied in each herd, taking into account the individual sensitivity and specificity of the tests. For this, specific computer software may be indispensable (e.g. the FreeCalc program $[10,11])$. Note that the average herd-level sensitivity will be $p$. 


\subsubsection{Number of herds to be studied}

Let $N$ be the total number of herds in the territory, $n$ the number of herds in the sample and $k$ the number of herds testing positive. The number $k$ will allow the one-tailed confidence interval to be calculated between zero and an upper limit ${ }^{1}$, within which there is a $95 \%$ or $99 \%$ probability of finding the true prevalence rate of infected herds. If the upper limit of the confidence interval lies below the prevalence rate threshold, there is at least a $95 \%$ (or $99 \%$ ) probability that the true prevalence rate of the disease in the population is below the set prevalence rate threshold (and that the territory qualifies for the status of territory where the disease is in the process of eradication).

If the prevalence rate threshold does not fall within the confidence interval, it may be considered that the prevalence rate of infected herds in the territory is below the threshold. If the prevalence rate threshold falls within the confidence interval, it cannot be stated that the prevalence rate of infected herds in the territory is below the threshold.

Example: if the prevalence rate threshold is set at $2 \%$ and the aim is to be $99 \%$ confident that the true prevalence is below this threshold, it will be seen that no positive responses must be obtained within at least 250 units to be able to decide that the prevalence is below the prevalence threshold. Given a sample of 500, the prevalence will

1. In practice, the necessary conditions for the classic calculation of the confidence interval for a percentage by an approximation of the binomial distribution using a normal distribution are rarely fulfilled. The upper limit of the one-tailed confidence interval at $(1-\alpha) \%$ can, however, always be calculated (even if $k=0$ ) using the approximation [28]:

$$
U_{L}=\frac{(k+1)}{k+1+\frac{n-k}{F_{1-\alpha}[2(k+1), 2(n-k)]}}
$$

where $F_{1-\alpha}[2(k+1), 2(n-k)]$ is the $(1-\alpha)$ percentile of a distribution $\mathrm{F}$ of degree of freedom $(2(k+1)$, $2(n-k))$. be considered to be below the threshold if fewer than 4 units in the sample give positive results.

The smaller the sample the greater the risk of coming to the wrong conclusion: either by concluding that the territory should not be considered in the process of eradication whereas in fact it could be, or by concluding that the territory may be considered as in the process of eradication whereas in fact it may not.

Thus, for a territory where the true prevalence rate is below the prevalence rate threshold, the smaller the sample, and the greater the risk of not concluding that the territory is in the process of eradication. The risk of obtaining an upper limit of the $95 \%$ one-tailed confidence interval that is above the prevalence rate threshold of $1 \%$ of herds, whereas the true prevalence rate in the territory is less than 1\%, is shown in Table $\mathrm{V}$. A similar table can be constructed for other prevalence rate thresholds $(0.5 \%, 0.2 \%$, etc.).

\subsubsection{Means of selecting herds}

As far as possible, these herds should be randomly selected from among the population in the territory.

\subsection{Disease-free territory}

Here, the question is fundamentally different. It is no longer a matter of evaluating (and thus quantifying) the level of prevalence, but rather of adopting a purely qualitative approach, so as to decide upon the presence or absence of the disease without having to test all the herds in the territory. It is therefore advisable to use the methods that will most effectively detect any outbreaks with the most appropriate confidence level (clinically expressed or latent infection), rather than giving an idea of the mean rate of herds infected. 
Table V. Probability of not being able to conclude that the prevalence rate of infected herds is less than $1 \%$, as a function of the true prevalence rate of infected herds in the territory and the sample size.

\begin{tabular}{llllllllllll}
\hline Sample size & $n$ & \multicolumn{10}{c}{ True prevalence in the territory $(\%)$} \\
\cline { 2 - 10 } & 0.01 & 0.05 & 0.1 & 0.2 & 0.3 & 0.4 & 0.5 & 0.6 & 0.7 & 0.8 & 0.9 \\
\hline
\end{tabular}

\section{fewer}

$\begin{array}{lrrrrrrrrrrrr}\text { than 300 }^{\text {a }} & & 100.0 & 100.0 & 100.0 & 100.0 & 100.0 & 100.0 & 100.0 & 100.0 & 100.0 & 100.0 & 100.0 \\ \mathbf{3 0 0} & 0 & 3.0 & 13.9 & 25.9 & 45.2 & 59.4 & 70.0 & 77.8 & 83.6 & 87.8 & 91.0 & 93.4 \\ \mathbf{5 0 0} & 1 & 0.1 & 2.7 & 9.0 & 26.4 & 44.2 & 59.5 & 71.3 & 80.2 & 86.5 & 90.9 & 94.0 \\ \mathbf{7 0 0} & 2 & 0.0 & 0.5 & 3.4 & 16.6 & 35.0 & 53.1 & 68.0 & 79.1 & 86.8 & 91.8 & 95.1 \\ \mathbf{8 0 0} & 3 & 0.0 & 0.1 & 0.9 & 7.9 & 22.1 & 39.8 & 56.7 & 70.7 & 81.0 & 88.2 & 92.9 \\ \mathbf{1 0 0 0} & 4 & 0.0 & 0.0 & 0.4 & 5.3 & 18.4 & 37.1 & 56.0 & 71.6 & 82.8 & 90.1 & 94.6 \\ \mathbf{1 , 2 5 0} & 6 & 0.0 & 0.0 & 0.0 & 1.4 & 8.6 & 23.8 & 43.4 & 62.2 & 77.0 & 87.1 & 93.2 \\ \mathbf{1 , 5 0 0} & 8 & 0.0 & 0.0 & 0.0 & 0.4 & 4.0 & 15.2 & 33.8 & 54.5 & 72.1 & 84.6 & 92.2 \\ \mathbf{2 , 0 0 0} & 12 & 0.0 & 0.0 & 0.0 & 0.0 & 0.9 & 6.3 & 20.8 & 42.4 & 64.2 & 80.8 & 90.9 \\ \mathbf{2 , 5 0 0} & 16 & 0.0 & 0.0 & 0.0 & 0.0 & 0.2 & 2.7 & 13.0 & 33.6 & 58.0 & 78.0 & 90.3 \\ \mathbf{5 , 0 0 0} & 38 & 0.0 & 0.0 & 0.0 & 0.0 & 0.0 & 0.0 & 0.6 & 6.4 & 27.0 & 58.4 & 83.5\end{array}$

The population is considered to be infinite (sampling fraction $<10 \%$ ). $n$ is the maximum number of positive results that can be obtained in the sample for the prevalence rate of infected herds to be considered as less than $1 \%$. Each percentage indicates the probability of obtaining a result greater than $n$ and consequently of not being able to conclude that the prevalence of infected herds in the territory is less than $1 \%$.

a With a sample of fewer than 300 units, the prevalence threshold of $1 \%$ will be within the $95 \%$ one-tailed confidence interval even if no positive results are obtained.

The approach will depend on whether one can identify sub-populations at a higher level of risk, in other words groups of individuals or herds with a higher probability of exposure, and thus a greater likelihood of being infected.

\subsubsection{Existence of sub-populations at a higher level of risk}

In this case, using a targeted sample, in other words covering only the sub-populations at higher risk can increase the probability of detecting the disease. The size of the sample within these sub-populations may vary. The probability of detecting the disease will of course increase with the size of the targeted sample. If the targeted sample includes only a part of the sub-population at higher risk, it can be chosen either randomly from within this sub-population or according to a risk gradient (for example, all the oldest animals in the case of diseases due to persistent infection).

\subsubsection{Absence of sub-populations at risk}

The sample will be selected randomly. The principle on which the sampling frame is based and how the results obtained will be interpreted must both be stated.

The choice of the prevalence rate threshold (e.g. $0.2 \%$ ), namely the minimum prevalence rate that one wishes to be able to detect, and the risk of obtaining a false negative result (e.g. $5 \%$ or $1 \%$ ) determine the sample size [27].

When the true, but unknown, prevalence rate is below the prevalence rate threshold, the risk of a false negative result will be correspondingly higher than the accepted risk ( $5 \%$ or $1 \%)$. However, there is a calculable and not inconsiderable probability of detecting (by means of a positive result) a prevalence rate markedly lower than the defined prevalence rate threshold. Thus, when the sample size has been defined with a 5\% risk of a false negative result, the probability of obtaining a positive response (and therefore of detecting the disease) ranges from $95 \%$ 
for a true prevalence rate equal to the prevalence rate threshold to $5 \%$ for a true prevalence rate some 58 times lower than the prevalence rate threshold. For a $1 \%$ risk the range of prevalence rates extends from the prevalence rate threshold to a prevalence rate 450 times lower. It should be borne in mind that the sample sizes thus determined allow the presence of the disease to be detected with a not inconsiderable probability for prevalence far lower than the one chosen.

After the survey has been implemented, the result obtained may be:

- No positive responses in the sample: this means that the disease is either absent from the territory or present at a prevalence rate below the set prevalence rate threshold (with a $5 \%$ or $1 \%$ risk of error).

- Positive responses in the sample: this confirms the presence of the disease in the territory (on condition that the test used has perfect specificity or after confirmation by an appropriate method) and does not allow the status "disease-free territory" to be accorded. An assessment needs to be made as to whether the situation corresponds to that of a "territory where the disease is in the process of eradication".

In the latter case it should be verified that the prevalence rate of the disease is below a prevalence rate threshold determined according to the characteristics of the disease. If the estimated rate is below this prevalence rate threshold, the chosen status should be "territory where the disease is in the process of eradication". If the estimated rate is above this prevalence rate threshold, the territory should be considered infected.

\subsubsection{Statistical relevance of previous data}

If the same survey is implemented periodically, the fact that negative results are repeatedly obtained suggests that the disease is either absent or that the prevalence rate is well below the prevalence rate threshold. The question is thus: "How to combine results from successive surveys?"

Assuming a totally stable epidemiological situation from one year to the next, the predictive value of the second survey could be enhanced by cumulating the data from the two surveys. In contrast, assuming a totally unstable epidemiological situation from one year to the next, the results of the first survey give, a priori, no information on the situation at the time of the second. The predictive value of the second survey must therefore be based purely on the results of this survey. Since the true situation will be somewhere between these two extremes (statistical independence or dependence), the diagnostic value of the second survey will in fact lie between: (a) the result of the second survey only and (b) the cumulated results of the two surveys. The statistician cannot give an exact value since this will depend on numerous factors.

A more general alternative is to evaluate the probability that a territory is infected by combining, using Bayes theorem, an a priori probability of the territory being infected, established before the protocol is applied, and the results following application of the protocol. The distribution of the a priori probability of infection will have to take into account a great number of factors that are not always easy to measure or combine: the results of previous tests, the quality of the Veterinary Services, inputs and controls on inputs, etc. The main problem with this very interesting approach lies in defining the probability (or distribution of probability), as objectively as possible.

One way in which previous data could be taken into account to substantiate a claim of disease-free territory would be for a study to be carried out, specific to the disease and to the control measures applied in the territory. Some recent studies put the base of such procedures $[6,7,25]$. A standardised procedure at the international level should be envisaged. 


\section{CONCLUSION}

Epidemiological surveillance for animal diseases and the end result, namely for a territory to achieve disease-free status, is becoming increasingly important. A global approach is needed. Once the general principles have been defined, the general requirements can be stated. Diseases should then be placed in categories based on epidemiological and clinical criteria, adapting or clarifying the general requirements for each category of disease.

When according a status care should be taken to use only terms that correspond exactly to the true or assumed animal health situation and are not open to confusion. In particular, the term "disease-free territory" should not be used for territories where outbreaks of infection or infestation persist. In such cases, more appropriate terms, such as "territory in which disease $\mathrm{X}$ is in the process of eradication" or "territory free from disease X in domestic animals", are preferable.

Statistical tools are an invaluable aid in determining sampling frames capable of ensuring the required level of epidemiological surveillance.

\section{ACKNOWLEDGEMENTS}

We would like to thank Moez Sanaa for his help in the redaction of the first version of this article, Thierry Chillaud for his critical reading of the text and helpful comments and Olivier Sparagano for his careful reading of the translation.

\section{REFERENCES}

[1] Acha P.N., Szyfres B., Zoonoses and Communicable Diseases to Man and Animals, 2nd ed., Office International des Épizooties, 1987, 1064 p.

[2] Anonymous, Council Directive 98/46/EEC of 24 June 1998 modifying the annexes of Directive $64 / 432 /$ EEC relating to problems of sanitary policing of inter-Community trade in animals of bovine and porcine species, 1998.
[3] Anonymous, Plague: Epidemiology, in: Center for control and prevention, Division of VectorBorne Infectious Diseases, URL: http://www. cdc.gov/ncidod/dvbid/plage. Page consulted on 13 September 2000.

[4] Anonymous, World Animal Health in 1999 , Office International des Épizooties, Paris, 2000, 2 volumes, $986 \mathrm{p}$.

[5] Aubert M.F.A., Costs and benefits of rabies control in wildlife in France, Rev. Sci. Tech. Off. Int. Epizoot. 18 (1999) 533-543.

[6] Audigé L., Doherr M.G., Salman M., A quantitative assessment of the validity of animal-health surveys using stochastic modelling, Prev. Vet. Med. 38 (1999) 259-276.

[7] Audigé L., Doherr M.G., Hauser R., Salman M.D., Stochastic modelling as a tool for planning animal-health surveys and interpreting screening-test results, Prev. Vet. Med. 49 (2001) 1-17.

[8] Bastian S., Buffereau J.P., Le Dream E., Bind J.L., Muller T., Toma B., La maladie d'Aujeszky en France en 1999, Épidémiol. Santé Anim. 37 (2000) 109-114.

[9] Bonjour P., Angot J.L., Dufour B., Sanaa M., Toma B., Une méthode quantitative d'évaluation des Services vétérinaires dans le cadre de l'analyse de risque à l'importation d'animaux vivants, Épidémiol. Santé Anim. 36 (1999) 39-63.

[10] Cameron A.R., Baldock F.C., A new probability formula for surveys to substantiate freedom from disease, Prev. Vet. Med. 34 (1998) 1-17.

[11] Cameron A.R., Baldock F.C., Two-stage sampling in surveys to substantiate freedom from disease, Prev. Vet. Med. 34 (1998) 19-30.

[12] Canon N.R., Audigé L., Denac H., Hofmann M., Griot C., Evidence of freedom from porcine reproductive and respiratory syndrome virus infected in Switzerland, Vet. Rec. 142 (1998) 142-143.

[13] Donald W.D., Gardner I.A., Wiggins A.D., Cutoff points for aggregate herd testing in the presence of disease clustering and correlation of test errors, Prev. Vet. Med. 19 (1994) 167-187.

[14] Dufour B., Méthode d'évaluation technicoéconomique de la qualité du fonctionnement des réseaux de surveillance épidémiologique des maladies infectieuses animales en vue de leur amélioration, Épidémiol. Santé Anim. 35 (1999) 11-20.

[15] Dufour B., La Vieille S., Epidemiological surveillance of infectious diseases in France, Vet. Res. 31 (2000) 169-185.

[16] Garner M.G., Gleeson L.J., Holyoaka P.K., Canon R.M., Doughty W.J., A national serological survey to verify Australia's freedom from porcine reproductive and respiratory syndrome, Aust. Vet. J. 75 (1997) 596-600.

[17] Hugh-Jones M., Keim P., Smith K., Coker P., Chromosomal genetic markers of Bacillus anthracis and related epidemiology, Proceedings of IXth International Symposium on Veterinary 
Epidemiology and Economics, Breckenridge, Colorado, 6-11 August 2000, 508-510.

[18] Jridi M., Bouzghaia M., Toma B., Maladie d'Aujeszky chez le sanglier en Tunisie, Épidémiol. Santé Anim. 30 (1996) 99-105.

[19] Jordan D., Aggregate testing for the evaluation of Johne's disease herd status, Aust. Vet. J. 73 (1997) 16-19.

[20] Martin S.W., Shoukri, M., Thorburn, M.A., Evaluating the health status of herds based on tests applied to individuals, Prev. Vet. Med. 14 (1992) 33-43.

[21] Moutou F., Dufour B., Savey M., Evaluation of the French foot-and mouth-disease epidemiovigilance network, Épidémiol. Santé Anim. 31-32 (1997) 07.08.1.

[22] Office International des Épizooties, International Animal Health Code, mammals, birds and bees. Paris, 9th ed., 2000, 484 p.

[23] Pérez-Sanchez R., Astigarraga A., Olega-Perez A., Encinas-Grandes A., Relationships between the persistence of African swine fever and the distribution of Ornithodoros erraticus in the province of Salamanca, Spain, Vet. Rec. 135 (1994) 207-209.

[24] Sanaa M., Use of detectable prevalence and acceptable prevalence concepts to declare a region or a country as "disease-free", Proceedings of
IXth International Symposium on Veterinary Epidemiology and Economics, Breckenridge, Colorado, 6-11 August 2000, 654-656.

[25] Schlosser W., Ebel E., Use of Markov-chain Monte Carlo model to evaluate the time value of historical testing information in animal populations, Prev. Vet. Med. 48 (2001) 167-175.

[26] Toma B., Vannier Ph., Eloit M., Aujeszky's disease, in: Manual of Standards for Diagnostic Tests and Vaccines, Office International des Épizooties, 3rd ed., 1996.

[27] Toma B., Dufour B., Sanaa M., Bénet J.J., Moutou F., Louza A., Ellis P., Applied veterinary epidemiology and the control of disease in populations, Association pour l'Étude de l'Épidémiologie et des Maladies Animales Ed., Paris, 1999.

[28] Wypij D., Binomial distribution, in: Armitage P., Colton T., Encyclopedia of biostatistics, Wiley \& sons, Chichester, 1998, pp. 359-363.

[29] Ziller M., Sclhorst T., Teuffert J., Schluter H., Sample strategies to substantiate freedom from disease: a theoretical approach, in: Proceedings of the Society for Veterinary Epidemiology and Preventive Medicine congress, Goodall E.A. and Thrusfield M.V. Eds., 24-26 March 1999, Bristol, UK, 1999, pp. 44-52. 